\title{
Analogical versus rule-based classification
}

\author{
WILLIAM D. WATTENMAKER, HEATHER L. MCQUAID, and STEPHANIE J. SCHWERTZ \\ University of Pittsburgh, Pittsburgh, Pennsylvania
}

\begin{abstract}
Sensitivity to feature co-occurrences was investigated as a function of analytic and analogical transfer. Participants memorized descriptions of hypothetical people and were then induced either to make transfer decisions by analogy to the descriptions (analogical transfer) or to search for and apply rules (analytic transfer). Across three experiments, analogical transfer was found to be more effective than analytic transfer for preserving co-occurring features in classification judgments. This result held for a variety of category structures and stimulus materials. It was difficult for subjects who adopted an analytic-transfer strategy to identify regularities that were embedded in stored instances. Alternatively, subjects who adopted an analogical-transfer strategy preserved feature co-occurrences as an indirect result of similarity-based retrieval and comparison processes. The effectiveness of analytic and analogical transfer is discussed.
\end{abstract}

There is clear evidence that different encoding strategies lead to differences in classification performance (e.g., Brooks, 1978, 1987; Kemler Nelson, 1984; Medin \& Smith, 1981; Nosofsky, Clark, \& Shin, 1989; Ward \& Scott, 1987; Wattenmaker, 1991, 1993). It seems likely that there are also a variety of transfer or postencoding strategies that can influence classification decisions, but previous research has only investigated influences of encoding strategies. Thus there is no direct evidence on how different postencoding strategies might influence classification. In the experiments described in this paper, the strategies that were used after encoding were varied, and relations between transfer strategies and classification performance were examined.

A central contrast in the experiments is between analytic and analogical transfer. Analytic transfer refers to a strategy of systematically analyzing stored information in order to develop explicit rules and generalizations. With analogical transfer, however, there is no attempt to form general rules. Instead, new instances are classified on the basis of their similarity to stored exemplars. Thus, with analogical transfer, items that are similar are classified together, whereas with analytic transfer, explicit rules are used to classify transfer items.

The distinction between analytic and analogical transfer is similar to the distinction between analytic and nonanalytic encoding (e.g., Allen \& Brooks, 1991; Brooks, $1978,1987)$ and to the distinction between intentional and incidental category learning (e.g., Kemler Nelson, 1984; Ward \& Becker, 1992; Wattenmaker, 1991, 1993). However, whereas these distinctions have been applied to

This research was supported by National Institute of Mental Health Grant MH45585 to W.D.W. We thank Lee Brooks, Jim Chumbley, Doug Medin, Ed Wisniewski, and four anonymous reviewers for helpful comments on an earlier draft of this manuscript. Correspondence concerning this article should be addressed to W. D. Wattenmaker, Division of Social Sciences, Widener University, 1 University Place, Chester, PA 19013. encoding processes, analytic and analogical transfer only refer to processes that occur after information has been encoded.

There has been a lot of research on analogical or exemplar-based transfer (e.g., Brooks, 1978; Estes, 1986; Hintzman, 1986; Kruschke, 1992; Medin \& Schaffer, 1978; Nosofsky, 1984, 1986, 1991; Ross, Perkins, \& Tenpenny, 1990; Wattenmaker, 1993; Whittlesea, 1987), but very little research on analytic transfer. For instance, if exemplars are stored, it has been assumed that decisions will be based on similarity relations between novel exemplars and stored exemplars (i.e., analogical transfer). An alternative possibility, however, is that the stored exemplars will be retrieved and analyzed, and that explicit rules will be formed (i.e., analytic transfer). The same type of hypothesis formation and testing that occurs with analytic encoding can occur after examples have been encoded. Indeed, it seems that people often need to retrieve and analyze past episodes. The success with which they can analyze and identify regularities in stored exemplars has not been directly investigated, however. ${ }^{1}$

One goal of the present experiments was to determine how effective analogical and especially analytic transfer would be for capturing complex regularities that were embedded in stored information. Analogical transfer can preserve regularities as a by-product of similarity-based retrieval. The multiplicative similarity assumption that is part of Medin and Schaffer's (1978) context model, for example, leads to the prediction that examples with correlated features should be especially likely to retrieve stored instances that also have these correlations. Indeed, there is evidence that analogical strategies can preserve complex regularities in classification (e.g., Brooks, 1978; Brooks \& Vokey, 1991; Medin, 1983; Medin, Altom, Edelson, \& Freko, 1982; Vokey \& Brooks, 1992; Wattenmaker, 1993).

In contrast to this research on analogical transfer, there is, as noted above, surprisingly little research that has investigated analytic transfer. Consequently, it is not 
clear how effective analytic transfer will be for capturing complex rules. One possibility is that limitations in working-memory capacity will make it difficult to search for and identify rules in stored exemplars (see Wattenmaker, 1992, for supporting evidence). Given the scarcity of data on analytic transfer, a main goal of this research was to investigate the effectiveness of analytic transfer. Note that the primary focus of the experiments was not to determine when these different strategies would be used, but rather to learn about the effectiveness with which analogical and especially analytic transfer can capture complex regularities.

A second goal was to examine the extent to which differences in classification would occur when different transfer strategies operated on identical representations. One possibility is that different transfer strategies will lead to large differences in classification performance. Alternatively, the content of the representation might be a powerful constraint on classification. Medin and Smith (1981), for example, varied the encoding strategies that were used to learn categories, and concluded that different strategies influenced the content of representations but not the categorization (i.e., transfer) processes that operated on these representations. Consistent with this possibility, Medin (1986) suggested that classification processes share fundamental commonalities, such as similarity-based retrieval of stored information, and that these commonalities will operate regardless of instructional variations. Indeed, Allen and Brooks (1991) found that similarity to stored exemplars influenced performance even in conditions in which subjects were given explicit rules and instructed to use these rules (see Nosofsky, 1991; Nosofsky et al., 1989, for further evidence in support of similarity-based retrieval processes). Thus, although there is clear evidence that different encoding strategies produce large differences in classification performance, it is less clear whether different postencoding strategies will produce large differences in classification.

\section{EXPERIMENT 1}

In this experiment, participants initially memorized a set of examples and were then induced to use either an analytic or an analogical strategy to make transfer decisions. The examples were divided into two categories on the basis of a complex rule involving feature co-occurrences, but the category assignments were concealed until the memorization task was completed. After the memorization task, the categories were revealed, the transfer strategies were induced, and a series of transfer tests was presented to examine the extent to which classification decisions in the analytic and analogical conditions would preserve the co-occurrences.

The learning examples that were used are shown in abstract notation in Table 1. There were two categories (Category A and Category B), and each category had four exemplars (A1-A4 in Category A and B1-B4 in Category B). Descriptions of hypothetical people were used to represent this structure, and each description had
Table 1

Abstract Notation of the Categories Presented in Experiment 1

\begin{tabular}{|c|c|c|c|c|c|c|c|c|c|c|c|}
\hline \multicolumn{12}{|c|}{ Category } \\
\hline \multicolumn{6}{|c|}{$\mathrm{A}$} & \multicolumn{6}{|c|}{ B } \\
\hline \multirow[b]{2}{*}{ Exemplars } & \multicolumn{5}{|c|}{ Dimensions } & \multirow[b]{2}{*}{ Exemplars } & \multicolumn{5}{|c|}{ Dimensions } \\
\hline & $\mathrm{DI}$ & $\mathrm{D} 2$ & D3 & D4 & D5 & & $\mathrm{D} 1$ & $\mathrm{D} 2$ & D3 & D4 & D5 \\
\hline Al & 1 & 1 & 1 & 1 & 1 & $\mathrm{Bl}$ & 1 & 0 & 1 & 0 & 0 \\
\hline A2 & 1 & 1 & 0 & 1 & 0 & $\mathrm{~B} 2$ & 1 & 0 & 0 & 1 & 0 \\
\hline A3 & 0 & 0 & 1 & 1 & l & B3 & 0 & 1 & 1 & 0 & 0 \\
\hline A4 & 0 & 0 & 0 & 0 & 1 & B4 & 0 & 1 & 0 & 0 & 1 \\
\hline
\end{tabular}

one feature on each of the five binary-valued dimensions (D1-D5) in Table 1 (e.g., for some subjects, Exemplar Al in Table 1 was represented by the description drives a blue car, was born in July, likes apples, drinks Diet Pepsi, and has light hair).

The values on the first two dimensions (D1 and D2) in Table 1 co-occurred. In Category A, whenever the value 1 occurred on $\mathrm{D} 1$, the value 1 also occurred on D2. Whenever the value 0 occurred on DI, however, the value 0 also occurred on D2. The opposite relations held for Category B: Whenever the value 1 occurred on D1, the value 0 occurred on $D 2$, and whenever the value 0 occurred on D1, the value 1 occurred on D2. A rule based on these co-occurring features would perfectly predict the category assignments of all the exemplars. Following Medin et al. (1982), the term correlation is used to describe this type of relation between features. Indeed, within the individual categories, the features are perfectly correlated. It is possible to capture this relationship by forming biconditional rules or rules consisting of disjunctions of conjunctions. This type of relationship between features has been studied extensively in the rule-learning and categorization literatures, and although this is a somewhat complex relation, there is evidence that people can detect regularities of this type without much difficulty if they are searching for rules during learning (e.g., Medin et al.,1982; Shepard, Hovland, \& Jenkins, 1961; Waldmann \& Holyoak, 1990; Wattenmaker, 1993). Of the six categories that Shepard et al. (1961) studied, for example, categories defined by this type of regularity were the second easiest to learn (also see Nosofsky, Gluck, Palmeri, McKinley, \& Glauthier, 1994). The values on D1, D2, and D3 occurred with equal frequency in both categories, but on D4 and D5, the value 1 was typical of Category A members, whereas the value 0 was typical of Category $B$ members.

The learning task was identical in the analogy and analytic conditions. Each example was randomly assigned a common male name (e.g., Dave: blue car, July, apple, Pepsi, light hair), and subjects memorized the features that corresponded to each of the names. The category assignments of the examples were not presented during memorization. When the memorization phase was completed, the names that had been associated with the exemplars were used to reveal the category assignments (e.g., "Dave, Jim, Steve, and Ed are in Category A, whereas Mike, Rob, Bill, and John are in Category B"). 
The descriptions that corresponded to these names were not presented either when the categories were revealed or at any time during the transfer phase. Thus analysis of the categories had to occur after the examples had been encoded.

Subjects in the rule (i.e., analytic) condition were instructed to analyze the stored exemplars and attempt to develop a rule or set of rules that would separate the descriptions in Category A from the descriptions in Category $\mathrm{B}$. In the analogy condition, to prevent subjects from attempting to develop rules, they were told that there were no rules that could be used to separate the categories. Instead of searching for rules, the subjects in the analogy condition were instructed to make classification decisions by deciding whether the transfer items seemed more like the individual descriptions in Category A or the individual descriptions in Category B.

The transfer items were designed to reveal the impact that the correlated features, feature typicality, and similarity relations had on classification. Sensitivity to the correlated features was examined by manipulating the features that occurred on the correlated dimensions (D1 and D2), and the use of feature typicality was examined by manipulating the features that occurred on the typical (D4 and D5) dimensions. The similarity relations between the transfer items and the learning examples were manipulated on the basis of the assumption that the number of learning examples that were highly similar to the test items might be a particularly important factor in classification. This should be especially true if subjects are using analogy. Indeed, many exemplar models (e.g., Brooks, 1978; Medin \& Schaffer, 1978; Nosofsky, 1984; Reed, 1972) assume that cases of high similarity will have a particularly strong impact on classification. The multiplicative-similarity assumption of the context model, for example, leads to the prediction that instances of high similarity should be especially important in classification (e.g., Medin \& Schaffer, 1978; Medin \& Schwanenflugel, 1981). In the present experiments, a learning example and a transfer item were defined as highly similar if they had the same features on four of the five dimensions. This measure of similarity has been useful for making predictions and explaining the results of a number of studies (e.g., Medin \& Schwanenflugel, 1981; Vokey \& Brooks, 1992; Wattenmaker, 1993).

Five different types of transfer test that varied the consistency between the correlation, feature typicality, and similarity were presented. The transfer tests that were presented are shown in abstract notation in Table 2. For the first type of test (Tests 1-4 in Table 2), the correlated features, the typicality of the features on the fourth and fifth dimensions, and cases of high similarity all predicted the same classification response. With the test item 11011 , for example, the values on the correlated dimensions (11---) occurred in Category A but never in Category B, and thus a Category A response would be consistent with the values on the correlated dimensions. Likewise, the value 1 on the fourth dimension and the value 1 on the fifth dimension are both more typical of
Table 2

Proportions of Responses That Were Consistent With the Correlated Features for the Transfer Tests in Experiment 1 as a Function of Whether Similaritv and Typicality Were Consistent (C), Neutral (N), or Inconsistent (I) With the Correlated Features

\begin{tabular}{|c|c|c|c|c|}
\hline \multirow[b]{2}{*}{ Transfer Test } & \multirow[b]{2}{*}{ Similarity } & \multirow[b]{2}{*}{ Typicality } & \multicolumn{2}{|c|}{ Condition } \\
\hline & & & Rule & Analogy \\
\hline 1. 11011 & $\mathrm{C}$ & $\mathrm{C}$ & .90 & .90 \\
\hline 2. 00011 & $\mathrm{C}$ & $\mathrm{C}$ & .80 & .90 \\
\hline 3. 01000 & $\mathrm{C}$ & C & .75 & .85 \\
\hline 4. 10000 & $\mathrm{C}$ & $\mathrm{C}$ & .80 & .80 \\
\hline \multicolumn{3}{|c|}{ Averaged proportions for Tests $1-4$} & .81 & .86 \\
\hline 5. 11000 & $\mathrm{C}$ & I & .50 & .65 \\
\hline 6. 00000 & $\mathrm{C}$ & I & $.43^{3}$ & .70 \\
\hline 7. 01011 & $\mathrm{C}$ & I & .45 & .90 \\
\hline 8. 10011 & $\mathrm{C}$ & I & .35 & .70 \\
\hline \multicolumn{3}{|c|}{ Averaged proportions for Tests $5-8$} & .44 & .74 \\
\hline 9. 11110 & $\mathrm{C}$ & $\mathrm{N}$ & .65 & .95 \\
\hline 10. 00101 & C & $\mathrm{N}$ & .45 & .90 \\
\hline 11. 11101 & $\mathrm{C}$ & $\mathrm{N}$ & .60 & .85 \\
\hline 12. 00110 & $\mathrm{C}$ & $\mathrm{N}$ & .50 & .75 \\
\hline 13. 10110 & $\mathrm{C}$ & $\mathrm{N}$ & .55 & .65 \\
\hline 14. 01101 & $\mathrm{C}$ & $\mathbf{N}$ & .55 & .80 \\
\hline 15. 10101 & $\mathrm{C}$ & $\mathrm{N}$ & .55 & .70 \\
\hline 16. 01110 & $\mathrm{C}$ & $\mathrm{N}$ & .70 & .65 \\
\hline \multicolumn{3}{|c|}{ Averaged proportions for Tests $9-16$} & .57 & .78 \\
\hline 17. 10111 & I & I & .40 & .25 \\
\hline 18. 01111 & I & I & .35 & .40 \\
\hline 19. 11100 & I & I & .40 & .30 \\
\hline 20. 00100 & I & I & .20 & .45 \\
\hline \multicolumn{3}{|c|}{ Averaged proportions for Tests $17-20$} & .34 & .34 \\
\hline 21. 01010 & I & $\mathrm{N}$ & .65 & .50 \\
\hline 22. 10001 & I & $\mathrm{N}$ & .45 & .40 \\
\hline 23. 11001 & I & $\mathbf{N}$ & .65 & .25 \\
\hline 24. 00010 & I & $\mathrm{N}$ & .40 & .50 \\
\hline \multicolumn{3}{|c|}{ Averaged proportions for Tests $21-24$} & .54 & .41 \\
\hline
\end{tabular}

Category A than of Category B examples. Finally, in terms of similarity, the transfer item 11011 is highly similar (i.e., differs on only one dimension) to two learning examples from Category A (11111 and 11010) but is not highly similar to any of the learning examples in Category B. Thus, a Category A response would also be consistent with the cases of high similarity.

For the second type of transfer test (see Tests 5-8 in Table 2), similarity was consistent with the correlation (i.e., predicted the same categorization response), but feature typicality was inconsistent with the correlated features (i.e., the use of the typical features on D4 and D5 would lead to a response that was inconsistent with the correlated features and with similarity). For the third type of transfer test (Tests 9-16), similarity and the correlation predicted the same response, but feature typicality was neutral (i.e., the feature on D4 and the feature on D5 made opposite predictions, so that overall feature typicality favored neither category). For the fourth test type (Tests 17-20), similarity and feature typicality were inconsistent with the correlated features, and for the fifth test type (Tests 21-24), similarity was inconsistent with the correlated features, while feature typicality was neutral. These tests were designed so that the results of 
the different test types could be compared with each other to assess the relative influence of the correlated features, feature typicality, and similarity in the rule and analogy conditions.

\section{Method}

Subjects. The subjects were 50 undergraduate students, who participated in the experiment to fulfill course requirements.

Stimuli and Procedure. Each description had one feature on each of the following dimensions: beverage preference (Diet Coke or Diet Pepsi), color of car (blue or green), month of birth (July or August), fruit preference (apples or peaches), and hair color (light or dark). To construct the descriptions from the notation in Table 1, each stimulus dimension (e.g., beverage preference) was assigned to one of the dimensions in Table ! (D1, D2, D3, D4, or D5), and the two features (c.g., Diet Coke vs. Diet Pepsi) on each of the stimulus dimensions were randomly assigned the value 1 or the value 0 . Five different assignments of dimensions and features were used, so that across subjects, each of the five stimulus dimensions occurred on all five dimensions (D 1-5) in Table 1 . The assignments and locations of dimensions were varied across subjects, but the dimensions were presented in a fixed order for each individual subject.

For the memorization task, the subjects were shown a sheet that listed the eight descriptions and the names that had been assigned to each of the descriptions, and they were given 20 minutes to memorize the names and the descriptions. The descriptions were listed in a random order, and each subject received a different randomization. At the end of this memorization phase, the names that corresponded to the descriptions were presented one at a time in a random order. When a name was presented, the subjects were instructed to write down all the characteristics that were associated with the name. Feedback was provided about the accuracy of recall after each recall attempt. After feedback had been provided, a new name was presented, and the procedure was repeated. After each run through the descriptions (i.e., after presentation of all eight names), the names were re-presented in a different order, and the recall and feedback procedures were repeated. The recallfeedback procedure continued until the subject either had two consecutive runs in which all of the features of each example were accurately recalled or until the allotted time for the recall-feedback phase $(30 \mathrm{~min}$ ) was exceeded. If the time limit was reached and recall on the final trial was $90 \%$ or higher (i.e., if the subject missed at most 2 of the 40 characteristics), the subject went on to the transfer phase, and the data were inciuded in the results. If a subject's recall was below this level, however, the data were excluded from further analysis. (Unless this criterion was reached, it would be difficult to distinguish between failures of encoding and failures of postencoding processes.) As described in the introduction to Experiment 1, the category assignments were presented after the recall phase. Participants had access to the category assignments throughout the transfer phase.

After the category assignments were presented, the subjects in the rule and analogy conditions were given the instructions described in the introduction to Experiment 1 . The first transfer test was presented after the transfer instructions were given. The subjects in both conditions were told that they could take as long as they wished to make transfer decisions. Those in the rule condition were told they could take as long as they liked to search for a rule before they started making transfer decisions. No writing was allowed during the transfer phase.

The 24 transfer tests in Table 2 were presented individually, and the subjects were instructed to indicate whether each example would be a better member of Category A or of Category B. The subjects in both conditions were encouraged to take as much time as they wanted to make their decisions. No feedback was provided during transfer, and the transfer tests were presented in a random order. After all of the items had been classified, the subjects were asked to explain (via written responses) the procedure they had used to make classification decisions, and to list the rules (if any) that they had used.

\section{Results}

Six subjects who were assigned to the rule condition and 4 subjects who were assigned to the analogy condition did not reach the recall criterion. The results of these subjects were excluded from further analysis. Twenty subjects in the analogy condition met the learning criterion; of these, recall on the last trial was $100 \%$ for $14,95 \%$ for 4 , and $90 \%$ for 2 . Likewise in the rule condition, 20 subjects met the learning criterion, and of these, recall on the last trial was $100 \%$ for $13,95 \%$ for 5 , and $90 \%$ for 2 .

Sensitivity to the correlated features can be assessed by examining the proportions of decisions that were consistent with the features on the correlated dimensions. For example, classifying a transfer item such as 11011 as a member of Category $\mathrm{A}$ would be consistent with the correlated features, but classifying it as a member of Category B would be inconsistent with the correlated features. Likewise, placing 01000 in Category B would be consistent with the correlation, but placing it in Category A would be inconsistent with the correlation. Across all the transfer tests, significantly more decisions were consistent with the correlated features in the analogy condition than in the rule condition [.65 vs. .54 ; $\left.F(1,38)=4.71, M S_{\mathrm{e}}=14.93, p<.05\right]$. Significantly more responses were consistent rather than inconsistent with the correlation in the analogy condition [.65 vs. $.35 ; t(19)=5.15, p<.001, S E=1.44]$, but in the rule condition, there was no significant difference between the number of responses that were consistent with the correlation and the number that were inconsistent with the correlation $[.54$ vs. $.46 ; t(19)=1.06, p=.30, S E=$ 1.96]. Thus, subjects in the analogy condition, but not those in the rule condition, tended to make responses that were consistent with the correlation.

Although the above results clearly show that classification decisions in the analogy condition tended to be consistent with the correlated features, the pattern of classifications that occurred on the five types of transfer tests indicated that this sensitivity derived from the use of similarity relations, rather than from direct access to correlations (see Table 2). Specifically, on the first three test types in Table 2, similarity was consistent with the correlated features, and on all three test types classifications were clearly consistent with the correlation $(.86, .74$, and .78$)$. On the last two test types in Table 2, however, similarity was inconsistent with the correlated features, and few classifications were consistent with the correlations (.34 and .41). In further support of the importance of similarity in the analogy condition, when typicality was neutral and similarity was inconsistent with the correlation (the last test type in Table 2), there was no significant difference between the number of responses that were consistent with the correlation and the 
number that were inconsistent with the correlation [.41 vs. . $59 ; t(19)=-1.44, p=.17, S E=.49]$; in fact, the results were in the opposite direction. In the following sections, the test types in Table 2 will be compared to determine the influence of similarity, typicality, and the correlation in the analogy condition and the rule condition.

The influence of similarity in the analogy and rule conditions. As noted earlier, the transfer tests were designed so that the test types in Table 2 could be compared with each other to assess the impact of similarity, typicality, and the correlation. The third and fifth test types and the second and fourth test types were designed to examine the influence of similarity. On the third test type (Tests 9-16) and the fifth test type (Tests 21--24), for example, typicality was neutral on all tests, but similarity was consistent with the correlation on the third test type and inconsistent with the correlation on the fifth test type. If similarity influenced decisions, more responses should be consistent with the correlation on the third test type than on the fifth test type. Comparison of these two test types revealed that when similarity switched from being consistent to being inconsistent with the correlation, there was a sharp decline in the proportion of responses that were consistent with the correlated features in the analogy condition (from .78 to .41). By contrast, there was a very slight decline in the rule condition on these test types (from .57 to .54 ). These results indicate that subjects in the analogy condition were much more sensitive to similarity relations than were subjects in the rule condition. Indeed, a 2 (condition) $\times$ 2 (test type) analysis of variance (ANOVA) revealed that this interaction between transfer condition and test type was highly significant $\left[F(1,38)=10.70, M S_{\mathrm{e}}=.059\right.$, $p<.01]$.

In addition, examination of the individual tests in these two test groups revealed that these results were highly consistent (see Table 2). In the analogy condition, for example, comparison of the 8 individual tests in the 9-16 group with the 4 individual tests in the 21-24 group reveals that every one of the 9-16 tests had a higher proportion of responses that were consistent with the correlation than did any of the individual tests in the 21-24 group. Thus, there was not a single individual test item for which the predicted pattern of results did not hold. Moreover, in almost all cases, the differences were very large. In contrast, in the rule condition, there was considerable overlap between the individual items in these two groups, and there were several cases in which higher proportions were observed for Tests 21-24 than for Tests 9-16.

These conclusions were also supported by the results of the second and fourth test types. On both of these test types, typicality was inconsistent with the correlation, but similarity was consistent with the correlation on the second test type (Tests 5-8) and inconsistent with the correlation on the fourth test type (Tests 17-20). Comparison of these tests revealed that when similarity switched from being consistent to being inconsistent with the correlation, there was a sharp decline in the proportion of responses that were consistent with the correlation in the analogy condition (from .74 to .34), but a relatively slight decline in this proportion in the rule condition (from .44 to .34). Again, this interaction between condition and test type shows that subjects in the analogy condition were much more sensitive to similarity relations $\left[F(1,38)=7.23, M S_{\mathrm{e}}=.92, p<.01\right]$. Examination of the individual transfer tests revealed that, again, the results were very consistent. In the analogy condition, each of the tests in the 5-8 group had a higher proportion of responses that were consistent with the correlation than did each of the 17-20 tests. In the rule condition, however, there were several cases in which higher proportions were observed for Tests 17-20 than for Tests 5-8.

The influence of typicality in the analogy and rule conditions. The first and third and the first and second test types were designed to reveal the influence of feature typicality. On the first test type (Tests 1-4) and the third test type (Tests 9-16), similarity was consistent with the correlation on all tests, but typicality was consistent with the correlation on the first test type and neutral on the third test type. The results of these tests showed that when typicality switched from being consistent with the correlation to being neutral, there was a large drop in the rule condition (from .81 to .57 ), but a relatively slight drop in the analogy condition (from .86 to .78 ). A 2 (condition) $\times 2$ (test type) ANOVA revealed that this interaction between learning condition and test type was significant $\left[F(1,38)=7.69, M S_{\mathrm{e}}=1.56, p<\right.$ $.01]$. This interaction indicates that subjects in the rule condition were more sensitive to differences in feature typicality than were subjects in the analogy condition. In addition, examination of the 4 individual transfer tests in the 1-4 group and of the 8 individual tests in the 9-16 group revealed that in the rule condition, each of the tests in which typicality was consistent with the correlation (i.e., Tests 1-4) had a higher proportion of responses that were consistent with the correlation than did each of the tests in which typicality was inconsistent with the correlation (i.e., Tests 9-16). Thus, again, the results were very consistent. In contrast, in the analogy condition, there were several cases in which higher proportions were observed for Tests 9-16 than for Tests $1-4$.

Examination of the first and second test types revealed this same interaction. When typicality switched from being consistent with similarity and the correlated features (the first test type) to being inconsistent with similarity and the correlation (the second test type), there was a sharp decline in the rule condition (from .81 to .44). Clearly, this result reflects extreme sensitivity to typicality in the rule condition. In contrast, there was a relatively slight decline in the analogy condition (from .86 to .74$)$. This interaction between condition and test type approached significance $\left[F(1,38)=3.80, M S_{\mathrm{e}}=\right.$ $1.31, p=.059]$. In addition, comparison of the individual tests in the 1-4 group with the individual tests in the 
5-8 group revealed that in the rule group, each of the tests in the 1-4 group had a higher proportion of responses that were consistent with the correlation than did each of the tests in the 5-8 group. In the analogy condition, however, in some cases, higher proportions were observed for the 5-8 group than for the 1-4 group.

The influence of the correlated features in the analogy and rule conditions. As noted above, when similarity was inconsistent with the correlation (i.e., in the fourth and fifth test types), no evidence of sensitivity to the correlated features was observed in the analogy condition. Given the clear use of feature typicality in the rule condition, the best way to examine the influence of the correlated features in the rule condition is by examining the results of the tests in which typicality was neutral (i.e., Tests 9-16 and Tests 21-24). An analysis of the number of responses that were consistent with the correlation versus the number of responses that were inconsistent with the correlation revealed that subjects in the rule condition showed little sensitivity to the correlated features on the fifth test type $[t(19)=.81, p=$ $.43]$ or on the third test type $[t(19)=1.19, p=.25]$.

Summary of the transfer tests. The transfer results indicate that subjects in the rule condition were primarily influenced by feature typicality, whereas subjects in the analogy condition were primarily influenced by similarity. Consistent with this conclusion, on the one test type in which similarity was pitted against typicality (i.e., the second test type in Table 2), large differences in classification behavior were observed between the analogy and rule conditions $\left[F(1,38)=9.12, M S_{\mathrm{e}}=1.58, p<\right.$ $.01]$. In addition, examination of the individual tests in the second group revealed that this result held for each of the individual tests, and that there were very large differences between almost all of the individual tests.

Strategy reports. To examine the influence that the transfer instructions had on the strategies that the subjects reported using, two raters independently classified the reports as describing an analogical strategy, a rulebased strategy, or some other strategy (the "other" category included strategies that were not rule or analogy based, that were highly idiosyncratic, that were too vague to understand, or cases in which a subject reported "guessing"). The raters agreed on $93 \%$ of the classifications. Disagreements were resolved by a third rater. ${ }^{2}$

The analysis of the reports indicated that the primary strategy that was used in the analogy condition consisted of classifying the examples on the basis of their similarity to stored instances (e.g., "I compared the description with each of the people in the categories, and looked for the person who was most similar to the description. I then placed the description in that category."). Fifteen subjects were classified as reporting this strategy. Three subjects, however, were classified as reporting the use of rules (e.g., "the people with a blue car were placed in Category $\mathrm{A}$ and the people with a green car were placed in Category B"). No subject in the analogy condition re- ported detecting or using the correlated features. The reports of 2 subjects were classified as "other."

An analysis of the data of the individual subjects as a function of the strategy they reported revealed clear differences in classification performance. Those subjects who reported using analogy made classifications that were consistent with the correlation $68 \%$ of the time, whereas those who reported using rules made classifications that were consistent with the correlation only $51 \%$ of the time. Thus, the advantage of using analogy over using rules was also evident in the data of individual subjects in the analogy condition.

In the rule condition, 14 subjects were classified as reporting the use of rules. To evaluate the accuracy of the rules that were formed, each rule was evaluated for how diagnostic it was of category membership. The diagnosticity of a rule was defined as being equal to the proportion of category exemplars that would be correctly classified by the rule minus the proportion of category exemplars that would be incorrectly classified by the rule. A rule based on the correlated features would be perfectly diagnostic, in that it would correctly classify all of the exemplars in Table 1 and lead to no incorrect classifications. Thus, it would have a diagnosticity of 1.0. Only 2 subjects in the rule condition reported this rule, however. Eight subjects reported using typical features (the diagnosticity of these rules was .50), and 4 subjects reported other rules that had little accuracy (the average diagnosticity of these rules was .06).

Two subjects in the rule condition were classified as reporting the use of analogy, and the reports of 4 subjects were classified as "other." Examination of the individual data of subjects in the rule condition revealed that subjects who had been classified as reporting the use of typical features, correlated features, other rules, or analogy made classification decisions that were consistent with the correlated features $46 \%$ of the time, $100 \%$ of the time, $51 \%$ of the time, and $58 \%$ of the time, respectively.

\section{Discussion}

Clear differences in classification behavior were observed as a function of transfer strategy. The results of the transfer tests and strategy reports indicated that classification decisions in the analogy condition were based on the similarity between the transfer items and stored instances, and the correlations were preserved in judgments as a by-product of this process. Indeed, there is evidence that correlated features might often be preserved in judgments as an indirect result of similaritybased retrieval (e.g., Medin, 1983; Medin et al., 1982; Wattenmaker, 1993). Since many regularities are expressed in the form of co-occurrences (e.g., Billman \& Heit, 1988; Medin et al., 1982; Rosch, 1978), it is adaptive to be able to preserve co-occurrences through analogical transfer.

Instead of using similarity relations or extracting the correlated features, the subjects in the rule condition pri- 
marily relied on differences in feature typicality. Although the rule involving the correlated features was perfectly predictive of category membership, the subjects in the rule condition tended to use simple rules that were less diagnostic than the correlation. Of the subjects who reported using a rule, $80 \%$ reported a rule that was based on a single dimension. These results suggest that it will be difficult to extract complex regularities from stored information with analytic transfer.

\section{EXPERIMENT 2}

Although little sensitivity to correlated features was observed in the rule condition of Experiment 1, the category structures might not have been well suited for detecting the correlations with analytic transfer. That is, when testing hypotheses about information in memory, people might initially search for simple rules that involve single dimensions, and only entertain more complex hypotheses if this search fails (e.g., Neisser \& Weene, 1962). Given limitations in working-memory capacity, the initial preference to seek simple rules might be even more pronounced with memory-based analyses. These considerations suggest that the typical features in Experiment 1 might have prevented subjects from entertaining more complex hypotheses involving feature co-occurrences.

To address this possibility, the category structures in Table 3 were used. These structures are similar to those used in Experiment 1 (e.g., D1 and D2 have correlated features), but the categories were designed so that all of the individual features and all of the two feature combinations (excluding the correlated features) occurred equally often in both categories. Thus, if simplicity is defined in terms of the number of logical operators (conjunction, disjunction, and negation) that are needed to perfectly predict category membership (see Neisser \& Weene, 1962; Nosofsky, 1991; Nosofsky et al., 1989), a rule involving the correlated features would be the simplest rule that could be used to partition the categories. The absence of typical features might increase the likelihood that these co-occurrences will be detected in the rule condition.

\section{Method}

Subjects. The subjects were 53 undergraduate students, who participated in the study to fulfill course requirements.

Table 3

Abstract Notation of the Categories Presented in Experiment 2

\begin{tabular}{|c|c|c|c|c|c|c|c|c|c|c|c|}
\hline \multicolumn{12}{|c|}{ Category } \\
\hline \multicolumn{6}{|c|}{$\mathrm{A}$} & \multicolumn{6}{|c|}{ B } \\
\hline \multirow[b]{2}{*}{ Exemplars } & \multicolumn{5}{|c|}{ Dimensions } & \multirow[b]{2}{*}{ Exemplars } & \multicolumn{5}{|c|}{ Dimensions } \\
\hline & D1 & $\mathrm{D} 2$ & D3 & D4 & $\overline{\mathrm{D} 5}$ & & $\overline{\mathrm{D} 1}$ & $\mathrm{D} 2$ & D3 & D4 & D5 \\
\hline A 1 & 1 & 1 & 1 & 0 & 0 & B1 & 1 & 0 & 0 & 0 & 1 \\
\hline A2 & 1 & 1 & 0 & 1 & 1 & $\mathrm{~B} 2$ & 1 & 0 & 1 & 1 & 0 \\
\hline A3 & 0 & 0 & 0 & 0 & 1 & B3 & 0 & 1 & 1 & 0 & 0 \\
\hline A4 & 0 & 0 & 1 & 1 & 0 & B4 & 0 & 1 & 0 & 1 & 1 \\
\hline
\end{tabular}

Table 4

Proportions of Responses That Were Consistent With the Correlated Features as a Function of Similarity for the Transfer Tests in Experiment 2

\begin{tabular}{ccc}
\hline \\
Transfer Test & Rule & Condition \\
\hline
\end{tabular}

Similarity Consistent with Correlated Features

$\begin{array}{lll}1 . & 11101 & .64\end{array}$

$\begin{array}{lll}2.11111 & .55 & .86\end{array}$

$\begin{array}{lll}3.11010 & .68 & .86\end{array}$

$\begin{array}{lll}\text { 4. } 00111 & .77 & .82\end{array}$

$\begin{array}{lll}5.00010 & .86 & .68\end{array}$

$\begin{array}{lll}6.00000 & .73 & .82\end{array}$

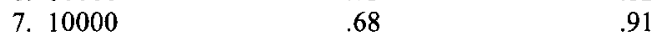

$\begin{array}{lll}8.10101 & .68 & .96\end{array}$

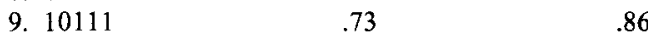

$\begin{array}{lll}10.01010 & .73 & .91\end{array}$

$\begin{array}{lll}11.01101 & .59 & .77\end{array}$

$\begin{array}{lll}12.01111 & .77 & .91\end{array}$

Averaged proportions

for Tests $1-12$

.70

.86

13. 11110

14. 00011

15. 10100

16. 01110

Similarity Neutral

Averaged proportions

for Tests 13-16

$\begin{array}{ll}.68 & .64 \\ .77 & .77 \\ .55 & .64 \\ .50 & .46 \\ & \\ .63 & .63\end{array}$

Stimuli and Procedure. The features and the procedure for constructing the descriptions were identical to those of Experiment 1 . The memorization phase, the transfer instructions, and the transfer phase were also identical to those of Experiment 1, except that the 16 transfer items in Table 4 were presented. For the first 12 tests in Table 4, similarity relations (as defined in Experiment 1) were consistent with the correlation, but on the last 4 tests in Table 4, similarity relations were neutral (i.e., favored neither category).

\section{Results}

Five subjects who were assigned to the rule condition and 4 subjects who were assigned to the analogy condition did not reach the recall criterion. Their results were excluded from further analysis. The results of the transfer tests are shown in Table 4 . When similarity was consistent with the correlation (Tests 1-12), the subjects in the analogy condition had a strong tendency to make classification decisions that were consistent with the correlated features. On these tests, the subjects in the analogy condition were much more likely to make responses that were consistent with the correlation than they were to make responses that were inconsistent with the correlation [.86 vs. $.14 ; t(21)=8.17, p<.01]$. This tendency was also observed in the rule condition [.70 vs. $.30 ; t(21)=3.76, p<.01]$. As in Experiment 1, however, the subjects in the analogy condition were significantly more likely to make classification decisions that were consistent with the correlation than were the subjects in the rule condition $\left[.86\right.$ vs. $.70 ; F(1,42)=5.08, M S_{\mathrm{e}}=$ $7.52, p<.05]$. This advantage for the analogy condition was observed for 11 of the 12 relevant tests in Table 4.

On the tests in which similarity was neutral (Tests 1316 ), in the analogy condition there was a sharp decline 
in the proportion of responses that were consistent with the correlation (from .86 to .63 ); in contrast, a relatively slight decline was observed in the rule condition (from .70 to .63 ). This decline was significant in the analogy condition $[t(21)=3.50, p<.01]$, but not in the rule condition $[t(21)=1.15, p=.26]$. Consistent with these observations, a 2 (condition) $\times 2$ (test type) ANOVA revealed a significant interaction between test type and learning condition $\left[F(1,42)=6.13, M S_{\mathrm{e}}=3.12, p<.02\right]$. This interaction indicates that subjects in the analogy condition were more sensitive to fluctuations in similarity than were subjects in the rule condition.

When similarity was neutral (Tests 13-16), no significant preference for the category that was consistent with the correlated features was observed in the analogy condition $[t(21)=1.71, p=.10, S E=.59]$. The direction of the results, however, suggests that there might have been some sensitivity to the correlation even when similarity was neutral. This trend was also observed in the rule condition, but again, this result was not reliable $[t(21)=1.91, p=.07, S E=.52]$.

Strategy reports. As in Experiment 1, the strategy reports were analyzed and classified as describing the use of rules or the use of analogy. The raters agreed on $96 \%$ of the classifications, and disagreements were resolved by a third rater. The reports indicated that it was still very difficult to detect the correlations in the rule condition. In fact, 11 subjects in the rule condition reported that they could not find any rules to separate the categories. Ten of these subjects reported that after they failed to identify rules, they looked for similarities between the test items and the individual learning examples (i.e., they spontaneously adopted an analogical strategy). Nine subjects in the rule condition did report identifying rules, but only 4 subjects correctly reported the correlation (the diagnosticity as defined in Experiment 1 was 1.0 for these rules). Five subjects reported other rules that had no validity (the diagnosticity of these rules was 0.0 ). The proportions of responses that were consistent with the correlation for subjects in the rule condition who reported using analogy, "other" rules, and the correlation were $.73, .57$, and 1.0 , respectively. Thus, the sensitivity to the correlation that was observed in the rule condition was due in large part to the subgroup of subjects who used analogy. The presence of so many analogy-oriented subjects in the rule condition also explains the decline in performance when similarity switched from being consistent to neutral with respect to the correlation (Tests $1-12$ vs. Tests $13-16$ in Table 4). In the analogy condition, all but 2 of the subjects were classified as reporting the use of analogy.

\section{Discussion}

Again, classification decisions in the analogy condition were more likely to preserve the correlated features than were classification decisions in the rule condition. As in Experiment 1, the subjects in the analogy condition made decisions by analogy to similar examples, and the co-occurrences were preserved as a by-product of this process. Even though there were no other regularities in the category structures, it was still very difficult to detect the co-occurrences in the rule condition. Many subjects in the rule condition spontaneously adopted an analogical strategy after they failed to identify rules, and the results of these subjects were very similar to the results of those subjects who were instructed to use analogy. Thus, rather than enabling subjects to detect the cooccurrences, the absence of simple rules encouraged subjects to adopt an analogical strategy.

\section{EXPERIMENT 3}

The exemplars in the first two experiments possessed no unique features, and exactly the same features occurred in several different examples (e.g., blue car, July, Diet Coke, light hair, apples vs. green car, July, Diet Coke, dark hair, apples vs. green car, July, Diet Pepsi, light hair, peaches, etc.). These examples are clearly very similar and easy to confuse. In addition to lacking distinctiveness, the exemplars were probably not well integrated (i.e., they consisted of five unrelated features), and they were not particularly familiar to the subjects. In Experiment 3, we examined the possibility that analytic transfer would be more effective for capturing co-occurrences if the exemplars were distinct, familiar, and well integrated.

Different stimulus materials were used to accomplish this. The stimuli were based on the following dimensions: having a connection with politics or entertainment; being male or female; and having a career that was active in the last six years or that has been inactive for several years. However, rather than following the usual practice of forming examples by presenting these features in different combinations (e.g., politics, male, active vs. politics, female, inactive vs. entertainment, female, active, etc.), a name of a familiar person who exemplified the underlying features was presented. For instance, rather than presenting the combination political, male, inactive, a familiar name such as Winston Churchill was presented. Likewise, rather than presenting the combination entertainer, male, inactive, a name such as Charlie Chaplin was presented. This procedure allowed the exemplars to be very distinct from each other, and also allowed the relevant features to be part of a well-integrated and very familiar representation. All of these factors should make it easier for postencoding analyses to be performed.

The abstract notation that was used to construct the examples is shown in Table 5. Although several of the abstract patterns re-occurred within a category (e.g., A2, $\mathrm{A} 3$, and $\mathrm{A} 4$ all had the same underlying structure), each occurrence was represented by a different person. Thus, if the pattern 111 corresponded to the features male, politician, inactive, A2 might be represented by Abraham Lincoln, A3 by Thomas Jefferson, and A4 by Winston Churchill.

The first two dimensions in Table 5 had correlated features (the Category A examples had 11 or 00 on these 
dimensions, whereas the Category $B$ examples had 10 or 01 ). To illustrate how these co-occurrences were expressed in the examples, assume that on D1 in Table 5, the value 1 corresponded to political, while the value 0 corresponded to entertainment; that on D2, 1 corresponded to male and 0 to female; and that on D3, 1 corresponded to inactive and 0 to active. Given this particular assignment, the first four Category A exemplars in Table 5 could be represented as Ted Kennedy, John Adams, Thomas Jefferson, and Winston Churchill, whereas Exemplars A5-A8 could be represented as Barbara Streisand, Judy Garland, Greta Garbo, and Rita Hayworth. In addition, given these dimensional assignments, Exemplars B1-B4 could be represented as Eleanor Roosevelt, Margaret Thatcher, Geraldine Ferraro, and Sandra O'Connor, while Exemplars B5-8 could be represented as Charlie Chaplin, Paul Newman, Robert DeNiro, and Michael Douglas. (Ratings were collected to ensure that the well-known people were perceived to have the correct combination of underlying features.)

Notice that the correlation between the first two dimensions is clearly expressed in the above examples and, indeed, it seems very salient. In Category A, all the males were connected to politics, whereas all the females were connected to entertainment. In Category B, however, all the females were connected to politics, whereas all the males were connected to entertainment. In addition to the correlated features, the value 1 on the third dimension was typical for Category A members, whereas the value 0 on the third dimension was typical for Category B members. A rule involving the correlated features was the simplest rule (in terms of the number of logical operators) that would perfectly partition the categories.

These stimuli would seem to place fewer demands on working memory and make it easier to analyze stored examples. In Experiments 1 and 2, for example, analyzing an exemplar required retrieving five separate features and keeping them active in working memory. With the current stimuli, however, if a name (e.g., Winston Churchill) is retrieved, all the relevant features can be easily and accurately retrieved or computed. The distinctiveness of the examples should also minimize confusions between examples during retrieval and analysis.

\section{Table 5}

Abstract Notation of the Categories Presented in Experiment 3

\begin{tabular}{|c|c|c|c|c|c|c|c|}
\hline \multicolumn{8}{|c|}{ Category } \\
\hline \multicolumn{4}{|c|}{ A } & \multicolumn{4}{|c|}{$\mathrm{B}$} \\
\hline \multirow[b]{2}{*}{ Exemplars } & \multicolumn{3}{|c|}{ Dimensions } & \multirow[b]{2}{*}{ Exemplars } & \multicolumn{3}{|c|}{ Dimensions } \\
\hline & $\mathrm{D} 1$ & D2 & $\overline{\mathrm{D} 3}$ & & $\overline{\text { D1 }}$ & D2 & $\overline{\text { D3 }}$ \\
\hline Al & 1 & 1 & 0 & B1 & 1 & 0 & $\overline{1}$ \\
\hline A2 & 1 & 1 & 1 & B2 & 1 & 0 & 0 \\
\hline $\mathrm{A} 3$ & 1 & 1 & 1 & B3 & 1 & 0 & 0 \\
\hline A4 & 1 & 1 & 1 & B4 & 1 & 0 & 0 \\
\hline A5 & 0 & 0 & 0 & B5 & 0 & 1 & 1 \\
\hline A6 & 0 & 0 & 1 & B6 & 0 & 1 & 0 \\
\hline A7 7 & 0 & 0 & 1 & B7 & 0 & 1 & 0 \\
\hline A 8 & 0 & 0 & 1 & B8 & 0 & 1 & 0 \\
\hline
\end{tabular}

Given the focus on postencoding analyses, the initial phase of the experiment consisted of a paired-associate learning task. Each person was randomly assigned a number between 1 and 16 (e.g., Winston Churchill-8), and the task was to memorize the name-number associations. When the name-number associations had been memorized, the numbers were used to introduce the categories (e.g., "the people who were assigned numbers 9 , $2,13,5,16,7,4$, and 10 are in Category $A$, while the people who were assigned numbers $14,1,15,11,8,6,3$, and 12 are in Category B"). The names were not presented when the categories were revealed and, consequently, as in the first two experiments, all analyses were based on exemplars that were not perceptually available. $^{3}$

Transfer tests were presented after the categories had been revealed. The transfer tests consisted of a list of three of the features that had been used to construct the examples (i.e., politics vs. entertainment, male vs. female, active vs. inactive), and the subjects classified these examples as more likely to belong in Category A or in Category B. The instructions in the analogy and rule conditions were the same as those in the earlier experiments, except that the subjects in the rule condition were told that they should attempt to form rules only in terms of the three underlying dimensions. The transfer tests were presented as lists of features, and the subjects were given the three relevant dimensions, to minimize the probability that idiosyncratic properties of the familiar people (e.g., nationality) would influence decisions and attempts to find rules.

Unique, familiar, and well-integrated materials should produce distinct representations of individual exemplars, which is likely to increase the accuracy of retrieval and the accessibility of the exemplars. Thus, these materials might increase the efficiency of analogical as well as analytic transfer. In either case, the results of the analytic and analogical transfer conditions will reveal whether the results of the first two experiments generalize to very different stimulus materials. In addition, to examine the transfer strategies that would be spontaneously used with these materials, a neutral condition was also tested. The subjects in this condition were not given any suggestions about the type of transfer strategy to use. As exemplar representations increase in distinctiveness, the probability of using analogical transfer is also likely to increase (Brooks, 1978, 1987; Jacoby \& Brooks, 1984). Thus, analogy might be a natural strategy to use with these materials.

\footnotetext{
Method

Subjects. The subjects were 96 undergraduates, who participated in the experiment to fulfill course requirements.

Stimuli. To ensure that the names would be perceived to be representative of the intended features, a separate group of 34 subjects was given names of well-known people and asked to indicate whether each person was male or female, connected to politics or entertainment, and active (i.e., had shown some activity in the last six years) or inactive (i.e., had shown no activity in the last six years) in their career. The subjects were also asked to indicate
} 
their confidence on a scale from $I$ (not very confident) to 5 (very confident). A name was only used if at least $85 \%$ of the subjects made the correct interpretation for all three dimensions. For the names that were used in the experiment, $98 \%, 100 \%$, and $96 \%$ of the subjects selected the correct features on the occupation, gender, and active dimensions, respectively. The average confidence ratings on these dimensions were $4.78,4.99$, and 4.41 , respectively. For some permutations (e.g., female, political, active), it was difficult to find names that were familiar enough to the subjects that the criterion could be reached on all of the dimensions. For each of the eight possible permutations of the three dimensions, however, at least four names were found that met the criterion on all dimensions. In the case of the inactive, male, entertainment combination, for example, the following names were used to represent this specific combination: Charlie Chaplin, Elvis Presley, Humphrey Bogart, Clark Gable, and John Wayne.

Six different arrangements of stimulus dimensions and features were used. The assignments of the stimulus dimensions (e.g., gender) were counterbalanced across the six orders, so that each stimulus dimension occurred equally often on all of the dimensions in Table 5. Across the six orders, an attempt was made to use all of the names that met the rating criterion. Thus, for one order, the combination inactive, male, entertainment might be represented as Charlie Chaplin, but in another order, this combination might be represented as John Wayne, and in a third order as Humphrey Bogart, and so on. The net effect of these procedures was that the names that were used to represent the structure in Table 5 were very different from order to order.

Procedure. During the memorization phase, the names were presented individually and the subjects attempted to recall the number that was associated with each name. (On the first run through the examples, the subjects had to guess the numbers that were associated with the names.) If the subject gave an incorrect response, the correct number was provided, and then the next name was presented. After each run through the 16 examples, the names were presented in a different order, and this procedure was repeated. The recall-feedback procedure continued until a subject had two consecutive errorless runs.

In terms of the abstract notation, the transfer items were 110 , 000,101 , and 011 . The transfer items consisted of the features that had been used to construct the examples (e.g., the transfer item 110 might be represented as entertainment; active; male). The four transfer items were presented twice, but the features were arranged in a different order for each presentation. Thus a total of eight classification decisions were made. The subjects in all conditions were told that if they were uncertain about whether a name possessed a certain feature (e.g., whether John Wayne was an entertainer), they could ask the experimenter.

Each of the transfer tests contrasted correlated features with feature typicality. With the test item 110 , for example, the features on the correlated dimensions (11-) were consistent with Category $\mathrm{A}$, but the feature on the typical dimension $(-0)$ was characteristic of Category B. Each learning example could be interpreted in terms of many features, of which the three manipulated features were only a subset. If only the dimensions that were manipulated are considered, however, each transfer item matched one of the learning examples in either Category A or Category B on the three underlying dimensions.

\section{Results}

In the analogy condition, across all of the transfer tests $78 \%$ of the classification decisions were consistent with the correlated features. Subjects in the neutral condition also showed a clear tendency to preserve the correlated features in classification, as $81 \%$ of the responses were consistent with the correlated features. In the rule condition, however, only $64 \%$ of the classifications decisions were consistent with the correlated features. Planned comparisons revealed that significantly more classifications were consistent with the correlated features in the analogy condition than in the rule condition $\left[F(1,62)=5.02, M S_{\mathrm{e}}=3.81, p<.05\right]$, and this was the case also for the neutral condition compared with the rule condition $\left[F(1,62)=7.78, M S_{\mathrm{e}}=3.71, p<.01\right]$. No significant difference was observed between the neutral and analogy conditions $\left[F(1,62)=.36, M S_{\mathrm{e}}=2.81\right.$, $p=.55]$.

As in Experiments 1 and 2, two raters classified each subject as reporting either an analogy-based or a rulebased strategy. The raters agreed on $96 \%$ of the classifications. Disagreements were resolved by a third rater. In the rule condition, 21 subjects were classified as reporting the use of rules and 10 subjects were classified as reporting the use of analogy. Of the subjects who reported using rules, only 5 accurately reported the correlation (the diagnosticity of the correlation was, again, 1.0); 11 reported inaccurate or low generality rules involving feature combinations (the average diagnosticity of these rules was .17), 3 reported inaccurate rules involving single features (the average diagnosticity was 0.0 ), and 2 reported using the typical features (the average diagnosticity was .50). The proportions of responses that were consistent with the correlation for subjects reporting the use of analogy, incorrect single features, feature combinations other than the correlated features, typical features, and the correlated features were $.80, .54, .56, .00$, and .98 , respectively.

In the neutral condition, the overwhelming majority of subjects reported retrieving individual learning examples that were similar to the test items (i.e., they spontaneously adopted an analogical strategy). Twenty-four subjects reported using analogy, while only 4 subjects reported using rules. Of those subjects who used rules, 3 reported inaccurate rules involving single features (the average diagnosticity was 0.0 ) and 1 subject correctly reported the correlation. The subjects in the neutral condition who reported using analogy, inaccurate rules, or the correlation made decisions that were consistent with the correlated features $80 \%$ of the time, $46 \%$ of the time, and $100 \%$ of the time, respectively.

The majority of subjects in the analogy condition also reported using analogy. Specifically, 25 subjects reported using analogy, while 3 subjects reported using inaccurate rules (the average diagnosticity of these rules was .08), and the reports of 4 subjects were classified as "other." The subjects who used analogy made decisions that were consistent with the correlated features $81 \%$ of the time, whereas the subjects who used rules made decisions that were consistent with the correlated features $63 \%$ of the time.

\section{Discussion}

Even though the stimuli in this experiment were designed to make it easier for postencoding analyses to be 
performed, few subjects in the rule condition detected the correlated features and, as in Experiments 1 and 2, classification decisions in the analogy condition were more likely to preserve the correlations than were decisions in the rule condition. Again, analogy preserved correlated features as a by-product of similarity-based retrieval processes. Thus, even though very different materials were used, the same results that occurred in Experiments 1 and 2 were observed. The vast majority of subjects in the neutral condition spontaneously adopted an analogical mode, and these subjects were as likely to make responses that were consistent with the correlation as were the subjects in the analogy condition.

It is important to note that in this experiment, as well as in Experiments 1 and 2, many subjects adopted analytic or analogical strategies when they were not instructed to do so. Thus, some subjects in the rule conditions used analogy, some subjects in the analogy conditions developed and applied rules, and subjects in the neutral condition used analogy or rules. The fact that subjects spontaneously adopted these modes clearly suggests that analytic and analogical strategies are natural strategies for people to use. It is also important to note that the results of the subjects who spontaneously adopted these strategies were entirely consistent with the results of the subjects who were instructed to use the strategies. Whether the subjects spontaneously adopted analogy or were encouraged by the instructions to use analogy, correlated features were preserved in their classification decisions. Likewise, whether subjects were instructed to search for rules or adopted this strategy on their own, the correlated features were rarely detected.

Generality of the results of Experiment 3. Even though the subjects were given the relevant features, it seemed possible that the idiosyncratic information (i.e., irrelevant features that were inherent in the names) might have made it difficult to detect the co-occurrences. To examine this possibility, we tested the ability of a separate group of 18 subjects to detect the correlation at encoding rather than at retrieval. The procedure for this experiment was identical to the procedure that was used in the rule condition of Experiment 3, except that the memory phase was replaced by a study phase in which the subjects were shown the names and the category assignments of the examples and given five minutes to develop rules.

The participants in this follow-up experiment had little difficulty detecting the correlated features. Indeed, $90 \%$ of the classification decisions were consistent with the rule, and $63 \%$ of the subjects explicitly reported using the correlation (in contrast, $15 \%$ of the participants in the rule condition of Experiment 3 reported the correlation). These results indicate that the correlated features were fairly easy to detect with these materials. Thus, the failure of subjects in the rule condition of Experiment 3 to detect the correlation appears to reflect limitations that are associated with memory-based analyses.
The follow-up study discussed above indicates that the presence of idiosyncratic information in the learning examples did not make it difficult to detect the cooccurrences. It is also possible, however, that the lack of idiosyncratic information in the transfer tests increased the effectiveness of analogical transfer. That is, the transfer items in Experiment 3 consisted of lists of relevant features, the features that participated in the correlation were expressed directly in this list, and unique or irrelevant features were not included in the transfer items. To examine whether analogical transfer can be effective under noisier retrieval conditions, we conducted an additional study in which the learning items and the transfer items possessed idiosyncratic information. Specifically, the learning examples, category structures, and procedures that were used in Experiment 3 were used in this follow-up experiment, but rather than presenting a list of features from the relevant dimensions as the transfer tests (e.g., inactive, male, politician), the names of people who represented these dimensions (e.g., Winston Churchill) were presented. Thus, in this experiment, the learning and the transfer items were represented by names of familiar people, whereas in Experiment 3 , only the learning items were represented by names of familiar people. The relevant dimensions were not presented during the learning or the transfer phase, and the transfer items were always new names. With the exception of the change in the nature of the transfer tests, this follow-up experiment was identical to Experiment 3.

With learning and transfer items of this type, there are many irrelevant or idiosyncratic properties that can be ascribed to the transfer items (nationality, political views, physical attributes, etc.). Thus, it seemed that these materials might decrease the influence that correlated features have on retrieval, but increase the influence that irrelevant features have on retrieval. Forty subjects were tested under analogical-transfer conditions, and the results indicated that analogical transfer can be effective even when the learning items and the test items have an abundance of idiosyncratic information. Across all of the transfer tests, the subjects were significantly more likely to make classifications that were consistent rather than inconsistent with the correlation [.60 vs. .40; $t(39)=2.24, p<.05]$.

In addition, analysis of the data of individual subjects revealed that 9 subjects reported using rules rather than analogy, and that only $32 \%$ of their responses were consistent with the correlation. In contrast, 27 subjects reported using analogy, and $73 \%$ of the responses of these subjects were consistent with the correlation. Thus, when analogy was used, it was very effective for preserving the correlated features. When rules were developed, however, they failed to capture the correlated features.

The results of this follow-up experiment indicate that analogy can be effective for preserving correlated features even when the correlations are not directly represented, and when both learning and transfer items have 
an abundance of idiosyncratic information. Although additional research is needed to determine the generality of this result, the results converge with the results of the first three experiments to suggest that analogical transfer can be effective for preserving co-occurrences in a variety of conditions.

\section{GENERAL DISCUSSION}

In all of the experiments, analogical transfer was more effective than analytic transfer for preserving feature cooccurrences. This result held across a variety of category structures and stimulus materials, and was highly consistent within as well as between conditions. Analogical transfer preserved the co-occurrences as a by-product of retrieval processes, but it was difficult to detect the cooccurrences with analytic transfer.

\section{The Effectiveness of Postencoding Analysis}

A clear result of these experiments was that subjects who used an analytic-transfer strategy were able to capture regularities involving single dimensions (e.g., in Experiment 1), but they had difficulty detecting feature co-occurrences. Even when the category structures (Experiment 2) and stimulus materials (Experiment 3 ) were designed to facilitate rule identification, little sensitivity to co-occurrences was observed.

Results that are very similar to the results that were observed in the analytic-transfer conditions have been found with memory-based sorting tasks (Wattenmaker, 1992). In these experiments, when the examples were visible during sorting, many types of interproperty relations (e.g., correlated features, causal relations, and family-resemblance principles) were used to form categories. When subjects attempted to form categories from memorized instances, however, there was a strong tendency to form categories around single dimensions rather than around interproperty relations. Even when it was possible to form categories on the basis of two perfectly correlated features (e.g., large and circular in one category and small and square in the other category), subjects in memory-based sorting conditions continued to form categories on the basis of a single dimension, whereas subjects in the perceptual sorting conditions showed a strong tendency to sort on the basis of the perfectly correlated features. Thus, it appears as if the results of the analytic-transfer conditions of the present studies will generalize to many different types of category structures and regularities.

The results of the analytic conditions indicate that the retrieval and computational processes that are required with analytic transfer make it difficult to develop accurate rules that involve features from multiple dimensions. Thus, postencoding processes that involve analytic transfer might often be of limited use for capturing complex regularities. The results of the analogical conditions, however, suggest that postencoding processes that are based on similarity and analogy can be more ef- fective for preserving complex regularities. Analogical transfer is adaptive because it provides a way for feature co-occurrences to be recovered and to influence classification decisions without overwhelming processing and memorial capacities.

Not only did the subjects in the rule conditions fail to identify the correlated features, the rules that were developed were often inaccurate or of limited validity. Given that the examples had been encoded and were represented in memory, it would not have been surprising if similarity-based retrieval and analogy had overpowered these weak rules. Rather than resorting to analogy, however, the subjects in the rule conditions tended to rely on the rules they developed. These results illustrate a limitation of rule-based transfer. When rules are developed, these rules appear to become more accessible than the exemplars upon which they are based, and consequently, the rules will override the input of other processes (e.g., similarity-driven retrieval of exemplars).

Although the consistency of the current results suggests that it will be difficult to detect complex regularities with analytic transfer, under some conditions analytic transfer might be fairly efficient. Consistent with this possibility, Wattenmaker (1991) found that when the processing goals are very specific and do not require extensive analysis, people can recover information about feature co-occurrences from stored examples (see Ross et al., 1990; Ross \& Spalding, 1991; and Spalding \& Ross, in press, for discussion of a mechanism for forming generalizations that involves stored instances but requires relatively little analysis). ${ }^{4}$

\section{Generality of the Results and \\ Relation to Other Work}

The advantages associated with analogical transfer did not depend on instructional manipulations; many subjects in all of the experiments spontaneously adopted analogical- or analytic-transfer strategies, and the advantages that were observed for analogy over analytic transfer also occurred with these groups of subjects. There are a number of considerations that support the generality of this result. As noted above, the available evidence suggests that it will often be difficult to capture complex regularities with analytic transfer. Analogy, however, has been shown to be effective for preserving complex regularities (e.g., Brooks, 1978; Brooks \& Vokey, 1991; Medin et al., 1982; Perruchet, in press; Vokey \& Brooks, 1992; Wattenmaker, 1993). In general, for analogy to be effective, it is important that novel items and stored instances that share a key regularity are perceived to be similar. In terms of co-occurring features, it seems that items that share co-occurring features will in fact often be perceived as being similar. In addition, if features combine in a multiplicative fashion (as predicted by the context model), correlated features might be especially likely to influence retrieval and similarity calculations (e.g., Medin, 1983; Medin et al., 1982; Medin \& Schaffer, 1978). ${ }^{5}$ More research is needed, 
however, to determine the types of regularities that are more likely to be preserved with analogical transfer than with analytic transfer.

It seems that both analogical and analytic transfer are common strategies that are used to make everyday decisions. In terms of analogical transfer, it is clear that people often make decisions on the basis of similarity to stored instances (e.g., Jacoby \& Brooks, 1984; Medin \& Ross, 1989). Although analytic transfer has received little attention, it seems that people often need to retrieve and analyze stored information to form rules or generalizations that will explain past events and enable current situations to be understood. For example, a clinician who encountered unexpected variability in the effectiveness of a treatment might retrieve and analyze successful and unsuccessful cases to determine the commonalities and differences between these cases. If a generalization or rule is formed, this rule can guide future treatment decisions. Likewise, we often need to analyze past experiences in terms of goals that were not active at encoding (e.g., questions such as what characterizes people you have known who have had financial difficulties, who have divorced, who have achieved great wealth, who have large families, who like pets, etc., involve, at least in part, retrieving and analyzing stored information in order to identify commonalities). The present results suggest that in many cases in which people attempt to form rules from stored instances, they might develop simplistic generalizations at the cost of missing more complex regularities that exist in stored information. ${ }^{6}$

Although analogical and rule-based transfer have been presented as distinct strategies, in some cases these strategies might interact. The subset of instances to be analyzed, for example, might be recruited on the basis of their similarity to the current situation. Analytic or ruleseeking activities might then operate on these retrieved examples. Indeed, this type of a strategy might avoid the errors that occur when extensive postencoding analyses are required, and also capitalize on the advantages that can accompany analogy. Whether analogical transfer, analytic transfer, or a combination of these strategies will be used to make decisions will probably depend on a number of factors, such as the distinctiveness of the exemplars, the similarity between current and past episodes, the nature of the activities that occurred at encoding, the availability of rules, the goals at retrieval (e.g., a need to be precise or to communicate conclusions might push people toward developing concise rules), and perhaps the cognitive style of the classifier.

The present experiments clearly show that different postencoding strategies can lead to large differences in classification performance. A major way in which learning strategies appear to affect classification is by influencing what is represented about a category (e.g., Medin, 1986; Medin \& Smith, 1981). Postencoding strategies might also influence classification by altering the information that enters into classification decisions. But whereas learning strategies exert their influence through selective encoding, postencoding strategies might alter the salience and accessibility of information that has already been encoded. Postencoding processes might change existing representations by adding new information to the representation (e.g., adding a set of rules to exemplar representations) or by altering the existing information (e.g., increasing the salience of some dimensions of stored examples while deemphasizing other dimensions; see Medin \& Edelson, 1988; Ross, 1989; and Ross et al., 1990, for related discussions).

As noted above, exemplar models have been successful in capturing a variety of regularities that occur in stimulus materials. The present results indicate that it is not only encoding operations, but the specifics of the retrieval processes that are crucially important for the success of exemplar models. The analytic- and analogicaltransfer conditions had identical encoding tasks in which intact exemplars were encoded, yet large differences occurred in classification. Analytic transfer resulted in the selective retrieval and use of one or two dimensions of the stored exemplars, whereas analogical transfer appeared to result in an influence of several dimensions. The latter retrieval process was much more effective for preserving structural regularities in classification. Thus, a retrieval process that permits input from several dimensions might be most effective for preserving complex regularities in classification judgments.

In summary, these experiments have shown that (1) the rules that are developed with analytic transfer will often be simplistic, or inaccurate, or have limited generality; (2) different transfer strategies can lead to large differences in classification performance; (3) analogical transfer can be more effective than analytic transfer for preserving complex regularities; and (4) analogy provides a mechanism for complex regularities to be retrieved and to influence classification decisions without overwhelming processing and memorial capacities.

\section{REFERENCES}

ALLEN, S. W., \& BROOKS, L. R. (1991). Specializing the operation of an explicit rule. Journal of Experimental Psychology: General, 120, 3-19.

Billman, D., \& HeIT, E. (1988). Observational learning from internal feedback: A simulation of an adaptive learning method. Cognitive Science, 12, 587-625.

BrooKs, L. R. (1978). Nonanalytic concept formation and memory for instances. In E. Rosch \& B. C. Lloyd (Eds.), Cognition and categorization (pp. 169-215). Hillsdale, NJ: Erlbaum.

BRoOKs, L. R. (1987). Decentralized control of categorization: The role of prior processing episodes. In U. Neisser (Ed.), Concepts and conceptual development: Ecological and intellectual factors in categorization (pp. 141-174). Cambridge: Cambridge University Press.

BROOKS, L. R., \& VOKEY, J. R. (1991). Abstract analogies and abst racted grammars: Comments on Reber (1989) and Mathews et al. (1989). Journal of Experimental Psychology: General, 120, 316-323.

EsTES, W. K. (1986). Array models for category learning. Cognitive Psychology, 18, 500-549.

HinTZMAN, D. L. (1986). "Schema abstraction" in a multiple-trace model. Psychological Review, 93, 411-428.

JACOBY, L. L., \& BROOKS, L. R. (1984). Nonanalytic cognition: Mem- 
ory, perception, and concept learning. In G. H. Bower (Ed.), The psychology of leaming and motivation: Advances in research and theory (Vol. 18, pp. 47-91). New York: Academic Press.

Kemler Nelson, D. G. (1984). The effect of intention on what concepts are acquired. Journal of Verbal Learning \& Verbal Behavior, 23, 734-759.

KRUSCHKE, J. K. (1992). ALCOVE: An exemplar-based connectionist model of category learning. Psychological Review, 99, 22-44.

MEDIN, D. L. (1983). Structural principles in categorization. In T. J. Tighe \& B. E. Shepp (Eds.), Perception, cognition, and development: Interactional analyses (pp. 203-230). Hillsdale, NJ: Erlbaum.

Meors, D. L. (1986). Comment on "Memory storage and retrieval processes in category learning." Journal of Experimental Psychology: General, 115, 373-381

Medin, D. L., Altom, M. W., Edelson, S. M., \& Freko, D. (1982). Correlated symptoms and simulated medical classification. Journal of Experimental Psychology: Human Learning \& Memory, 8, 37-50.

Medrn, D. L., \& Edelson, S. (1988). Problem structure and the use of base rate information from experience. Journal of Experimental Psychology: General, 117, 68-85.

Medin, D. L.. \& Ross, B. H. (1989). The specific character of abstract thought: Categorization, problem-solving, and induction. In R. J. Sternberg (Ed.), Advances in honor of William K. Estes (Vol. 2, pp. 207-234). Hillsdale, NJ: Erlbaum.

MEdin, D. L., \& SCHAFFER, M. M. (1978). Context theory of classification learning. Psychological Review, 85, 207-238.

Medin, D. L., \& Schwanenflugel, P. J. (1981). Linear separability in classification learning. Journal of Experimental Psychology: Human Learning \& Memory, 7, 355-368.

Medin, D. L., \& SMITH, E. E. (1981). Strategies and classification learning. Journal of Experimental Psychology: Human Learning \& Memory, 7, 241-253

NEISSER, U., \& WEENE, P. (1962). Hierarchies in concept attainment. Journal of Experimental Psychology, 64, 640-645.

NosofSKY, R. M. (1984). Choice, similarity, and the context theory of classification. Journal of Experimental Psychology: Learning, Memory, \& Cognition, 10, 104-114

Nosofsky, R. M. (1986). Attention, similarity, and the identificationcategorization relationship. Journal of Experimental Psychology: General, 115, 39-57.

NosOFSKY, R. M. (1987). Attention and learning processes in the identification and categorization of integral stimuli. Journal of Experimental Psychology: Learning, Memory, \& Cognition, 13, 87-109.

NosofsKy, R. M. (1991). Typicality in logically defined categories: Exemplar-similarity versus rule instantiation. Memory \& Cognition, 19, $131-150$.

Nosofsky, R. M., Clark, S. E., \& ShIN, H. J. (1989). Rules and exemplars in categorization, identification, and recognition. Journal of Experimental Psychology: Learning, Memory, \& Cognition, 15, 282-304.

Nosofsky, R. M., Gluck, M. A., Palmeri, T. J., McKinley, S. C., \& Glauthier, P. (1994). Comparing models of rule-based classification learning: A replication and extension of Shepard, Hovland, and Jenkins (1961). Memory \& Cognition, 22, 352-369.

Perruchet, P. (in press). Learning from complex rule-governed environments: On the proper functions of nonconscious and conscious processes. In C. Umiltà \& M. Moscovitch (Eds.), Attention and performance XV: Conscious and nonconscious information processing. Cambridge, MA: MIT Press.

REED, S. K. (1972). Pattern recognition and categorization. Cognitive Psychology, 3, 382-407.

Rosch, E. (1978). Principles of categorization. In E. Rosch \& B. B. Lloyd (Eds.), Cognition and categorization (pp. 27-48). Hillsdale, NJ: Erlbaum.

Ross, B. H. (1989). Remindings in learning and instruction. In S. Vosniadou \& A. Ortony (Eds.), Similarity and analogical reasoning (pp. 438-469). Cambridge: Cambridge University Press.

Ross, B. H., Perkins, S. H., \& Tenpenny, P. L. (1990). Remindingbased category learning. Cognitive Psychology, 22, 460-492.

Ross, B. H., \& SPalding, T. L. (1991). Some influences of instance comparisons in concept formation. (Tech. Rep. No. CS-91-03). Urbana, IL: University of Illinois.
Shepard, R. N., Hovland, C. I., \& Jenkins, H. M. (1961), Learning and memorization of classifications. Psychological Monographs, 75 (13, Whole No. 517)

SPaldiNG, T. J., \& Ross, B. H. (in press). Comparison-based learning: Effects of comparing instances during category learning. Journal of Experimental Psychology: Learning, Memory, \& Cognition.

VOKEY, J. R., \& BROOKS, L. R. (1992). Salience of item knowledge in learning artificial grammars. Journal of Experimental Psychology: Learning. Memory. \& Cognition, 18, 328-344.

WaldmanN, M. R., \& Holyoak, K. J. (1990). Can causal induction be reduced to associative learning? In Proceedings of the Twelfth Annual Conference of the Cognitive Science Society (pp. 190-197). Hillsdale, NJ: Erlbaum.

WARD, T. B., \& BECKER, A. H. (1992). Learning categories with and without trying: Does it make a difference? In B. Burns (Ed.), Percepts, concepts, and categories (pp. 451-491). New York: Elsevier.

WARD, T. B., \& SCOTT, J. (1987). Analytic and holistic modes of learning family-resemblance concepts. Memory \& Cognition, 15, 42-54.

WATTENMAKER, W. D. (1991). Learning modes, feature correlations, and memory-based categorization. Journal of Experimental Psychology: Learning Memory, \& Cognition, 17, 908-923.

WATTENMAKER, W. D. (1992). Relational properties and memorybased category construction. Journal of Experimental Psychology: Learning. Memory, \& Cognition, 18, 1125-1138.

WATTENMAKER, W. D. (1993). Incidental concept learning, feature frequency, and correlated properties. Journal of Experimental Psychology: Learning, Memory, \& Cognition, 19, 203-222.

WhitTlesea, B. W. A. (1987). Preservation of specific experiences in the representation of knowledge. Journal of Experimental Psychology: Learning, Memory, \& Cognition, 13, 3-17.

\section{NOTES}

1. It should be noted that some exemplar models have mechanisms that can produce rule-like representations. The Medin and Schaffer (1978) context model, for example, has a selective encoding mechanism that allows for large differences in the salience of dimensions (also see Nosofsky, 1986, 1987, 1991; Nosofsky et al., 1989; and Wattenmaker, 1993). The idea that is emphasized here, however, is that exemplar models do not explicitly acknowledge the possibility that subjects will engage in a process of searching for rules after encoding. That is, these models assume that the salience of dimensions can be altered by processes that occur during encoding, but they do not explicitly allow for the possibility that dimensional salience can be altered by hypothesis-testing activities that occur after encoding

2. Any report that described using a feature or feature combination to represent ali or several members of a category was classified as rule based, whereas any report that described comparing the test items to individual learning examples was classified as analogy based. Thus, reports that were classified as rule based provided general statements about category membership (e.g., "people who had blue cars were in Category A and people who had green cars were in Category B"), whereas subjects who were classified as using analogy did not provide general statements about category membership, but instead reported comparing the transfer items to individual learning instances.

3 . The transfer tests in Experiments 1 and 2 required retrieving experimentally learned name-to-feature associations. Thus, the namenumber paired associate learning procedure makes this task comparable to the first two experiments in that the subjects in the present experiment also needed to retrieve experimentally learned associations in order to perform the transfer task.

4. Specifically, Ross et al. (1990) proposed that if commonalities between a retrieved instance and a novel item are noticed, these commonalities can be abstracted and represented as explicit generalizations. It is important to note, however, that this type of a remindingbased abstraction mechanism is different in many respects from the analytic-transfer strategies that were investigated in the present experiments. For example, generalizations that are formed with reminding-based learning are essentially a by-product of an analogicaltransfer strategy, whereas generalizations that are formed with analytic-transfer strategies are the product of explicit and systematic attempts to retrieve and analyze instances. 
5. The exact predictions of the context model depend on the salience of the dimensions, the structure of the categories, and the properties of the transfer tests. In this regard, it should be noted that the use of analogy, whether based on instructions or spontaneously adopted, would not necessarily preserve the feature co-occurrences in these experiments. For example, analogy might not have preserved the cooccurrences if subjects relied on only one or two highly accessible examples, if the noncorrelated dimensions were highly salient, if partial exemplars were retrieved, if there was selective attention to a few dimensions of the transfer items, or if subjects placed a lot of weight on cases of medium similarity between learning and transfer tests.
6. $1 \mathrm{t}$ is also worth noting that it seems that many types of regularities in the environment are as complex as, or considerably more complex than, those studied in the present experiments. Indeed, many cat egories have examples that possess opposite values on correlated dimensions (e.g., a rapid heart beat with high blood pressure or a very slow heart beat with low blood pressure can both suggest a cardiovascular ailment; likewise, eating a lot and sleeping a lot or eating very little and sleeping very little can both suggest depression).

(Manuscript received August 17, 1993; revision accepted for publication June 30,1994 .)

\section{Cognitive Science Program National Institute of Mental Health}

The Cognitive Science Program of the National Institute of Mental Health (NIMH) invites grant applications for basic research in such areas as perception, action, attention, memory, learning, knowledge, reasoning, and language. Methods may be drawn from psychology, artificial intelligence, linguistics, philosophy, and neuroscience. Research may be conducted with humans or other animals, at any stage in the lifespan. Purely computational work is supported, but it should make substantive contact with behavioral or physiological evidence.

Funding mechanisms include grants for research projects (of various sizes and durations), institutional training grants, pre- and postdoctoral fellowships, career scientist awards, and conference grants. Grants are awarded primarily, but not solely, to institutions in the U.S. Individual inyestigators need not be U.S. citizens; however, recipients of pre-/postdoctoral fellowships and career scientist awards must be U.S. nationals or permanent residents.

General information and application kits for NIMH grants are available at most U.S. institutions' research offices. (Information is also available electronically via gopher.nimh.nih.gov.) Further questions can be directed to

Howard S. Kurtzman, Ph.D.

Chief, Cognitive Science Program

National Institute of Mental Health

5600 Fishers Lane, Rm. 11C-16

Rockville, MD 20857

(e-mail: kurtzman@nih.gov; phone: 301-443-9400; fax: 301-443-4822) 